\title{
76. ÉVOLUTION SÉCULAIRE DES ORBITES DE PARTICULES MÉTÉORIQUES
}

\author{
J. DELCOURT
}

Centre National d'Etudes des Télécommunications, Issy-les-Moulineaux, France

\begin{abstract}
We study the secular evolution of the orbit described by a meteoric particle moving under the influence of planetary perturbations and the Poynting-Robertson effect. Using a simplified model, this study is made with only one disturbing planet. We present preliminary results on the long-term variation of the osculating elements of the orbit.
\end{abstract}

\section{Introduction}

Une liaison radioélectrique a permis d'observer les traînées ionisées créées par des particules météoriques dont la magnitude limite, estimée d'après la sensibilité de l'appareillage est de l'ordre de $M=10$. L'étude présentée est faite conjointement avec le dépouillement de ces observations.

\section{Hypothèses}

(1) On ne considère qu'une seule planète perturbatrice, Jupiter. Dans cette étude préliminaire, son orbite est supposée circulaire et fixe.

(2) La particule météorique est supposée sphérique de diamètre $d \gg \lambda$, longueur d'onde du rayonnement incident; elle absorbe toute l'énergie incidente et la rayonne d'une manière isotrope. La force de Poynting-Robertson s'exprimera à l'aide d'un scalaire $K$.

(3) On néglige l'influence éventuelle du rayonnement corpusculaire du Soleil, qui pourrait être représenté par des équations analogues à celles de l'effet PoyntingRobertson.

(4) On néglige ici l'influence possible d'une charge électrique acquise par la particule.

\section{3. Équations du Mouvement}

Soit un trièdre d'inertie $T^{\prime}$, d'origine $S$; plan $x^{\prime} S y^{\prime}$ : orbite de Jupiter $J$. L'accélération totale agissant sur la particule $P$ est

$$
\begin{aligned}
\mathbf{\Gamma}_{T} & =-\frac{G m_{0}}{r^{3}} \mathbf{r}+\frac{K c}{r^{3}}\left(1-\frac{\dot{r}}{c}\right) \mathbf{r}-\frac{K}{r^{2}} \mathbf{V}+G m^{\prime}\left(\frac{\rho}{\rho^{3}}-\frac{\mathbf{r}^{\prime}}{r^{\prime 3}}\right) \\
& =-\frac{\mu^{\prime}}{r^{3}} \mathbf{r}+\mathbf{\Gamma} \\
\mathbf{r} & =-K \frac{\dot{r}}{r^{3}} \mathbf{r}-\frac{K}{r^{2}} \mathbf{V}+\mathbf{\Gamma}_{J}
\end{aligned}
$$

où $m_{0}, m^{\prime}$ sont les masses du Soleil $S$, de Jupiter $J ; \mathbf{r}=\mathbf{S P}, \mathbf{r}=\mathbf{S J}, \boldsymbol{\rho}=\mathbf{r}^{\prime}-\mathbf{r} ;$ et $\mathbf{V}$ est la vitesse de $P$ dans $T^{\prime}$. 
On décompose $\boldsymbol{\Gamma}$ et $\boldsymbol{\Gamma}_{J}$ suivant les composantes radiale, perpendiculaire et binormale; respectivement $\left(R^{*}, S^{*}, W^{*}\right)$ et $(R, S, W)$. On obtient

$$
R^{*}=-2 K \frac{\dot{r}}{r^{2}}+R, \quad S^{*}=-K \frac{\dot{v}}{r}+S, \quad W^{*}=W,
$$

où $v$ est l'angle polaire de $P$ sur son orbite.

\section{Variations Séculaires des Éléments}

On utilise la méthode de Gauss: on applique l'opérateur moyenne double en $M$ et $M^{\prime}$ au système différentiel en $a, e, i, \Omega, \omega, \varepsilon$ et on obtient

$$
\begin{aligned}
& \dot{\bar{a}}=-K \frac{2+3 e^{2}}{a\left(1-e^{2}\right)^{3 / 2}}+\frac{2 e}{2 \pi n} \int_{0}^{2 \pi} \bar{R} \sin E \mathrm{~d} E+\frac{2 \sqrt{1-e^{2}}}{n} \frac{1}{2 \pi} \int_{0}^{2 \pi} \bar{S} \mathrm{~d} E \\
& \dot{\bar{e}}=-K \frac{5 e}{2 a^{2}\left(1-e^{2}\right)^{1 / 2}}+\frac{1-e^{2}}{2 \pi n a} \int_{0}^{2 \pi} \bar{R} \sin E \mathrm{~d} E+\frac{\left(1-e^{2}\right)^{3 / 2}}{2 \pi n a e} \int_{0}^{2 \pi} \bar{S} \mathrm{~d} E \\
& -\frac{\sqrt{1-e^{2}}}{2 \pi n a e} \int_{0}^{2 \pi} \bar{S}(1-e \cos E)^{2} \mathrm{~d} E \\
& \dot{\bar{\imath}}=\frac{\cos \omega}{2 \pi n a \sqrt{1-e^{2}}} \int_{0}^{2 \pi} \bar{W}(\cos E-e)(1-e \cos E) \mathrm{d} E \\
& -\frac{\sin \omega}{2 \pi n a} \int_{0}^{2 \pi} \bar{W}(1-e \cos E) \sin E \mathrm{~d} E \\
& \dot{\bar{\Omega}}=\frac{\sin \omega}{2 \pi n a \sqrt{1-e^{2}} \sin i} \int_{0}^{2 \pi} \bar{W}(\cos E-e)(1-e \cos E) \mathrm{d} E \\
& +\frac{\cos \omega}{2 \pi n a \sin i} \int_{0}^{2 \pi} \bar{W}(1-e \cos E) \sin E \mathrm{~d} E \\
& \dot{\bar{\omega}}=-\frac{\sqrt{1-e^{2}}}{2 \pi n a e} \int_{0}^{2 \pi} \bar{R}(\cos E-e) \mathrm{d} E+\frac{1-e^{2}}{2 \pi n a e} \int_{0}^{2 \pi} \bar{S} \sin E \mathrm{~d} E \\
& +\frac{1}{2 \pi n a e} \int_{0}^{2 \pi} \bar{S}(1-e \cos E) \sin E \mathrm{~d} E-\dot{\bar{\Omega}} \cos i \\
& \dot{\vec{\varepsilon}}=-\frac{2}{2 \pi n a} \int_{0}^{2 \pi} \bar{R}(1-e \cos E)^{2} \mathrm{~d} E+\dot{\bar{\varpi}}\left(1-\sqrt{1-e^{2}}\right) \\
& +2 \dot{\Omega \Omega} \sqrt{1-e^{2}} \sin ^{2} \frac{i}{2} \text {. }
\end{aligned}
$$


où

$$
\bar{R}=\frac{1}{2 \pi} \int_{0}^{2 \pi} R \mathrm{~d} M^{\prime},
$$

et également $\bar{S}$ et $\bar{W} ; \tilde{\omega}=\Omega+\omega, \epsilon=\tilde{\omega}-n \tau$.

\section{Méthode de Gauss-Halphen}

Cette méthode est utilisée pour calculer les composantes $\bar{R}, \bar{S}, \bar{W}$ de $\bar{\Gamma}_{J}$. On en trouve un exposé succinct par Musen (1963).

De l'expression de $\boldsymbol{\Gamma}_{J}$, on tire aisément

$$
\overline{\boldsymbol{\Gamma}}_{J}=\frac{G m^{\prime}}{2 \pi} \int_{0}^{2 \pi} \frac{\rho}{\rho^{3}} \mathrm{~d} M^{\prime} .
$$

Le lieu de $\rho$ est un cône elliptique de sommet $P$. On démontre que

$$
\overline{\boldsymbol{\Gamma}}_{J}=-\frac{G m^{\prime}}{\pi a^{\prime 2} z^{\prime}} \boldsymbol{\Phi} \cdot \mathbf{r}
$$

avec

$$
\Phi=\frac{1}{2} \int_{E^{\prime}} \frac{\rho}{\rho^{3}}(\rho \times \mathrm{d} \rho) ; \quad\left(E^{\prime}: \text { orbite de Jupiter }\right) .
$$

$\boldsymbol{\Phi}$ ne dépendant pas de $|\rho|, E^{\prime}$ peut être remplacée par une section quelconque du cône. Le problème est alors de déterminer les composantes de $\boldsymbol{\Phi}$ et de $\mathbf{r}$ dans un trièdre approprié. En particulier, en prenant un trièdre $P x y z$ de sommet $P$, coïncidant avec les axes principaux du cône et en prenant pour courbe d'intégration la section par un plan $z=1$, les 3 composantes diagonales non nulles de $\boldsymbol{\Phi}$ s'expriment, à l'aide des intégrales elliptiques complètes de Legendre, à partir des coordonnées de $P$ dans $T^{\prime}$. Mais la détermination des axes principaux d'un cône de sommet et de base connus demande la résolution d'une équation du 3e degré (méthode de GaussHill). Halphen a montré que l'on peut éviter cette résolution. On prend pour contour d'intégration la biquadratique gauche, intersection du cône et de la sphère centrée sur $P$, de rayon unité (courbe unicursale de paramètre $s$ ) et on fait le changement de variable $s \rightarrow u$ :

$$
\sqrt{(s-A)(s-B)(s-C)}=-\frac{1}{2} p^{\prime} u,
$$

où $A, B, C$ sont les grandeurs des axes principaux.

La méthode est résumée dans les points suivants (Halphen, 1886):

(1) les composantes de $\Phi$ s'expriment en fonction de la période réelle $\omega$ de $p u$ et de $\eta=\zeta \omega$ (où $p u, \zeta u$ sont les fonctions elliptiques de Weierstrass).

(2) $\omega$ et $\eta$ s'expriment en fonction des invariants elliptiques $g_{2}$ et $g_{3}$ de $p u$.

(3) le changement de variables $s \rightarrow u$ relie les fonctions symétriques des racines de $p u$, soit $g_{2}$ et $g_{3}$ et les fonctions symétriques des racines de l'équation en $s$ du cône (trois invariants non nuls du cône). 
(4) ces invariants du cône s'obtiennent eux-mêmes à partir d'une configuration donnée du cône, c'est-à-dire finalement, en fonction des coordonnees de $P$ dans $T^{\prime}$. Ainsi, on aboutit à

où

$$
\overline{\boldsymbol{\Gamma}}_{J}=-\Psi \cdot \mathbf{r},
$$

$$
\Psi=A \mu+B \nu,
$$

et $\mu, \nu$ sont des dyadiques symétriques dont les composantes sont des polynômes respectivement de degrés 6 et 2 suivant les coordonnées de $P$ (dans $\left.T^{\prime}\right)$. $A$ et $B$ sont des scalaires dont les expressions sont:

$$
\begin{aligned}
B & =\frac{4}{\pi} g_{2}^{-5 / 4}\left[K F\left(\frac{1}{12}, \frac{5}{12}^{5} ; \frac{1}{2} ; \xi\right)-\left(\operatorname{sgn} g_{3}\right) \frac{\pi}{4 \sqrt{3} K} \xi^{1 / 2} F\left(\frac{7}{12}, \frac{11}{12} ; \frac{3}{2} ; \xi\right)\right] \\
B & =\frac{4}{\sqrt[4]{12}} g_{2}^{-5 / 4}\left(\frac{1+\sqrt{\xi}}{2}\right){ }^{-1 / 6} F\left(\frac{1}{6}, \frac{1}{6} ; 1 ;-\frac{1-\sqrt{\xi}}{1+\sqrt{\xi}}\right), \quad g_{3}>0 \\
A & =+\left(\operatorname{sgn} g_{3}\right) \frac{40}{3 \pi \sqrt{3}} K g_{2}^{-11 / 4} \xi^{1 / 2} F\left(\frac{1}{1} \frac{3}{2}, \frac{1}{1} \frac{7}{2} ; \frac{3}{2} ; \xi\right)-\frac{8}{K^{\prime}} g_{2}^{-11 / 4} F\left(\frac{7}{12}, \frac{11}{12} ; \frac{3}{2} ; \xi\right) \\
& -\frac{154}{27 K} g_{2}^{-11 / 4} \xi F\left(\frac{1}{1} \frac{9}{2}, \frac{2}{1} \frac{3}{2} ; \frac{5}{2} ; \xi\right)
\end{aligned}
$$

$$
A=-\frac{10}{9} \sqrt[4]{12} g_{2}^{-11 / 4}\left(\frac{1+\sqrt{\xi}}{2}\right)^{-7 / 6} F\left(\frac{7}{6}, \frac{1}{6} ; 2 ;-\frac{1-\sqrt{\xi}}{1+\sqrt{\xi}}\right), \quad g_{3}>0
$$

où $\xi=27 g_{3}^{2} g_{2}^{-3}$ est l'invariant de Klein, $K=K(1 / \sqrt{2})=\Gamma^{2}\left(\frac{1}{4}\right) / 4 \sqrt{ } \pi$, où $K(k)$ est l'intégrale elliptique complète de deuxième espèce, et $F(\alpha, \beta ; \gamma ; z)$ est la fonction hypergéométrique de Gauss.

Apparemment, Musen ne considère pas le cas où $g_{3}<0$. Les formules (2) qu'il utilise ne sont valables que pour $g_{3} \geqslant 0$; d'ailleurs, elles proviennent des développements au voisinage de $\xi=1$ des solutions de l'équation hypergéométrique de Bruns, et de ce fait, convergent très lentement au voisinage de $\xi=0$, c'est-à-dire précisément lorsque $g_{3}$, passant par 0 , change de signe. Nous avons montré qu'au voisinage du plan de Jupiter, $g_{3}$, considéré comme fonction du rayon polaire $r$ de $P$, devient négatif pour $a^{\prime} / \sqrt{ } 2<r<a^{\prime} \sqrt{ } 2$ ( $a^{\prime}$ étant le rayon de l'orbite de $J$ ).

\section{Effet Poynting-Robertson}

L'influence du seul effet Poynting-Robertson sur les orbites des très petits corps a été étudiée (Robertson, 1937; Wyatt et Whipple, 1950). Avec les hypothèses du (2) on montre que

$$
K=7.1 \times 10^{-8} d^{-1} \delta^{-1} \mathrm{UA}^{2} \mathrm{an}^{-1},
$$

$\delta$ étant la densité de la particule. Les calculs ont été effectués avec plusieurs valeurs de $K$; en particulier $K=2 \times 10^{-6} \mathrm{UA}^{2}$ an $^{-1}$ (Figures 1 à 5 ) correspond à une particule sphérique, de densité $\delta \approx 3$ et de diamètre $d \approx 100 \mu \mathrm{m}$. 


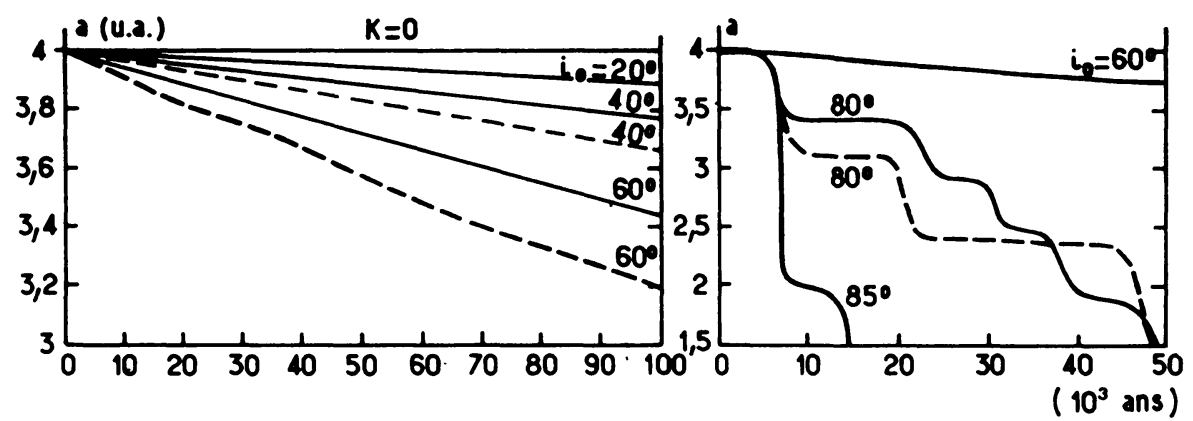

Fig. 1. Variation de $a\left(a_{0}=4 ; e_{0}=0,4\right.$ et 0,$\left.6 ; \omega_{0}=90^{\circ}\right)$.

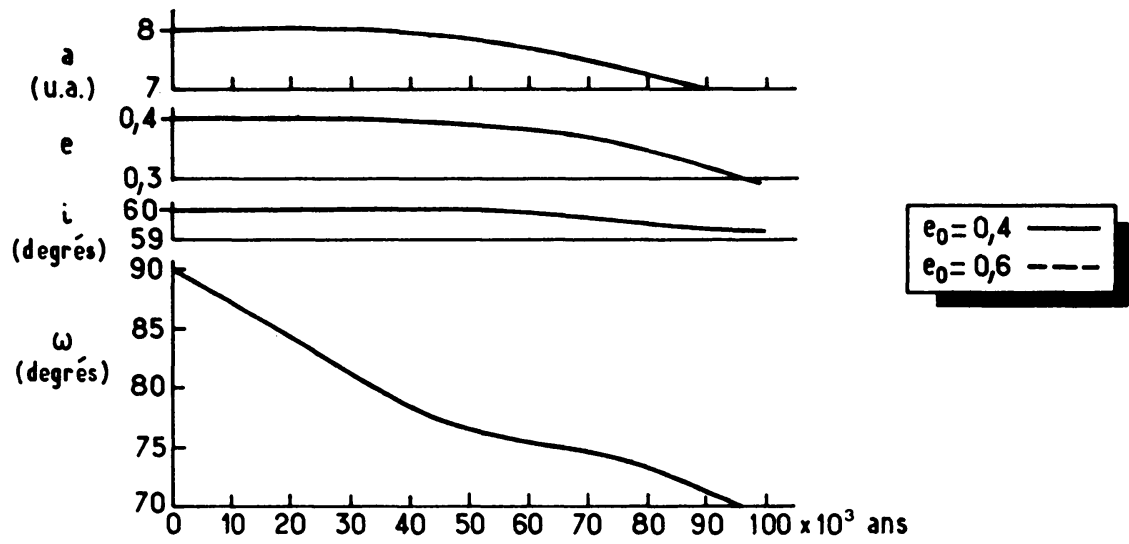

Fig. 2. Variation de $a, e, i$ et $\omega\left(a_{0}=8 ; e_{0}=0,4 ; i_{0}=60^{\circ}, \omega_{0}=90^{\circ}\right)$.

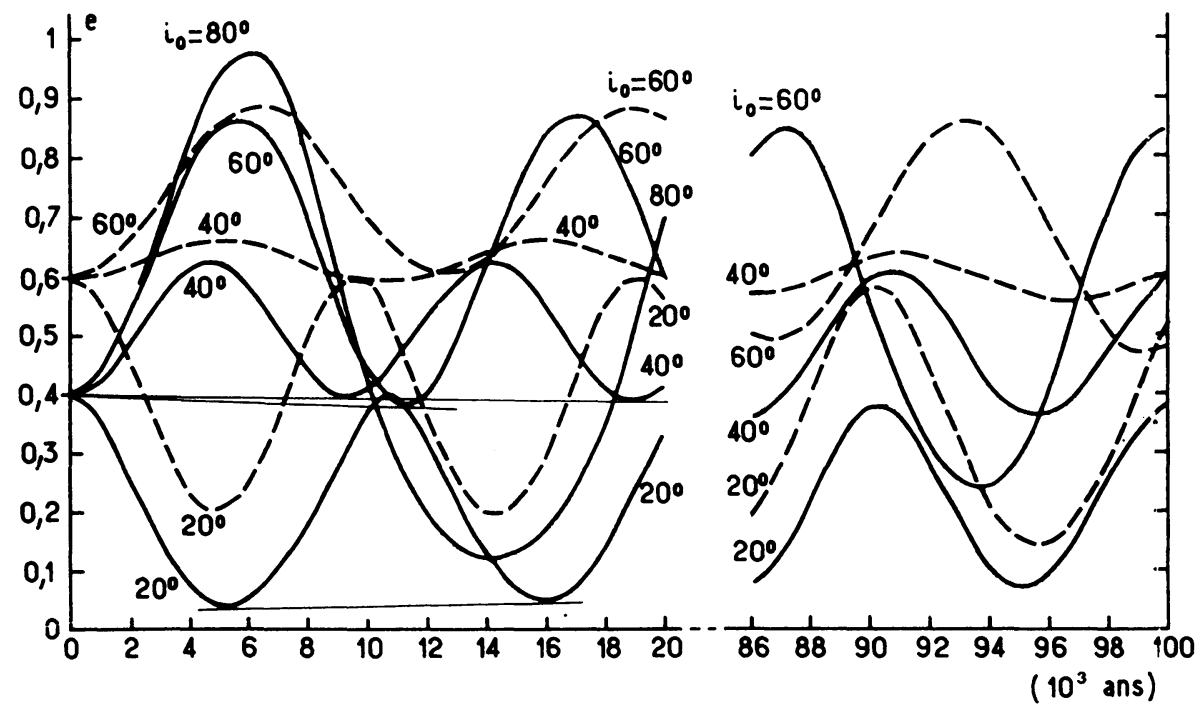

Fig. 3. Variation de $e\left(a_{0}=4 ; e_{0}=0,4\right.$ et 0,$\left.6 ; \omega_{0}=90^{\circ}\right)$. 


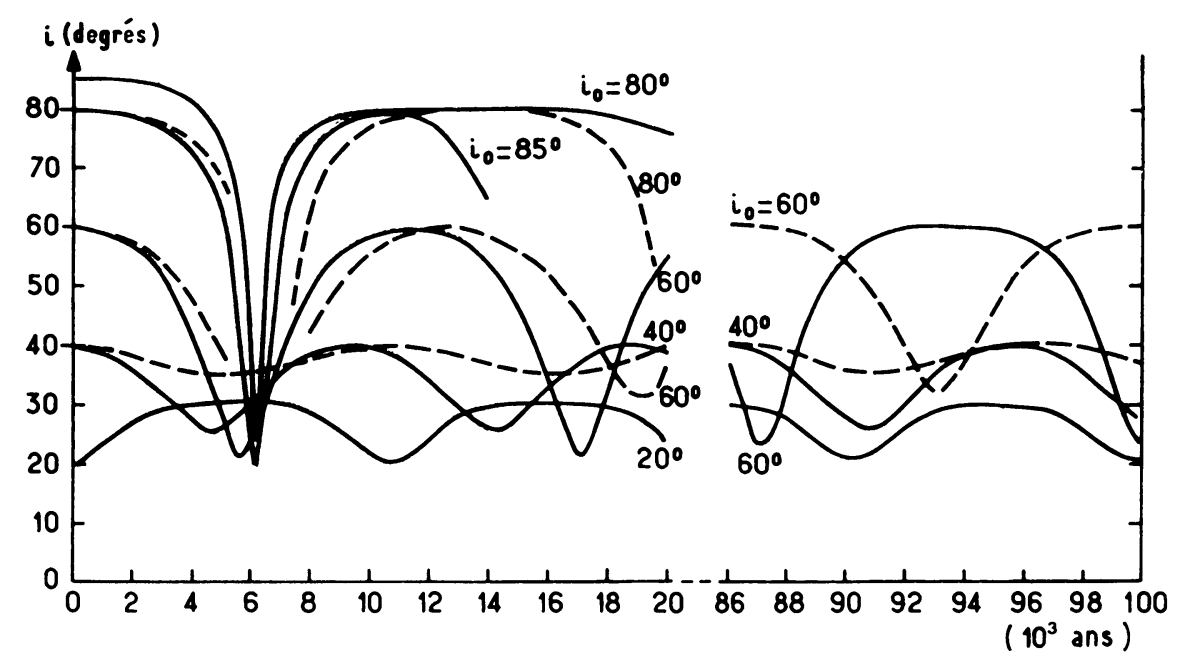

Fig. 4. Variation de $i\left(a_{0}=4 ; e_{0}=0,4\right.$ et 0,$\left.6 ; \omega_{0}=90^{\circ}\right)$.

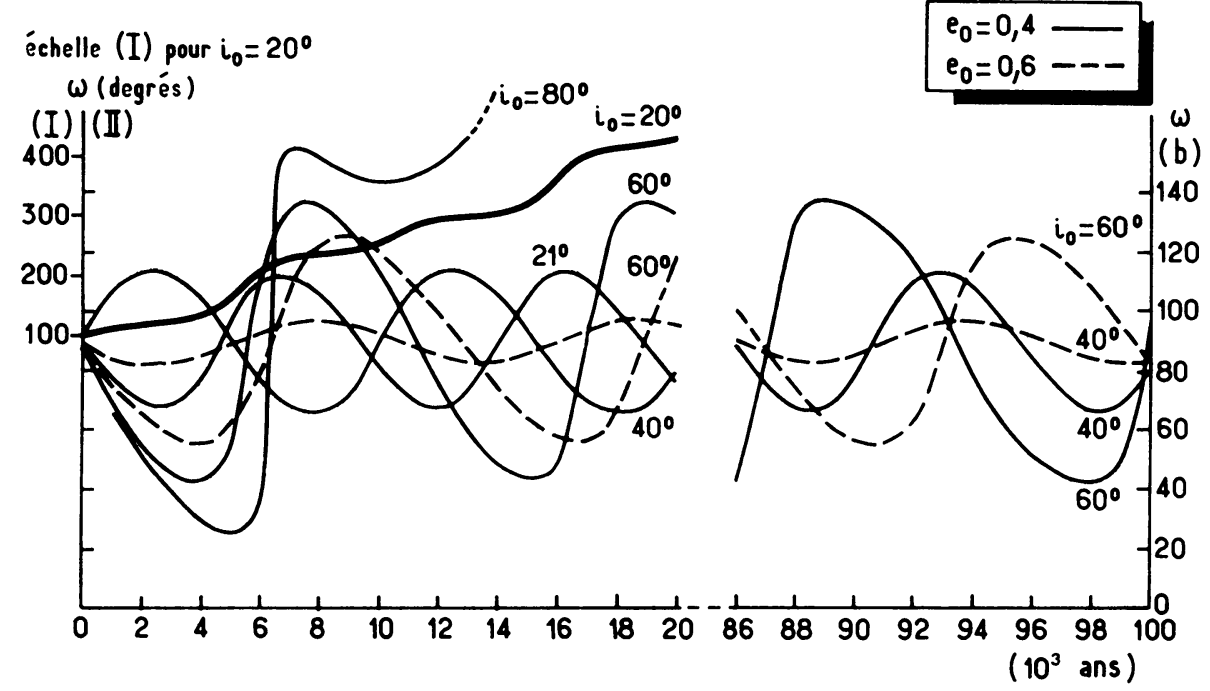

Fig. 5. Variation de $\omega\left(a_{0}=4 ; e_{0}=0,4\right.$ et 0,$\left.6 ; \omega_{0}=90^{\circ}\right)$.

\section{Intégration Numérique}

Le système différentiel moyenné en $M$ et $M^{\prime}$ est intégré numériquement (sur calculateur C II-10070) par une méthode à pas liés. On utilise un algorithme proposé par Hamming (1959), dérivé de l'algorithme de Milne, qui a une stabilité analogue et permet, en général, d'obtenir un ordre plus élevé de l'erreur de troncature, soit, dans le cas présent, $\mathrm{O}\left(h^{6}\right), h$ étant le pas d'intégration.

Un test de proximité des deux orbites est appliqué en permanence; toute approche 
en deçà d'une certaine limite choisie (par example, 0.3 UA) est signalée et constitue dans le programme actuel, un point d'arrêt de la solution. Une étude plus complète sur ce sujet est en cours.

\section{Résultats}

Les calculs ont été effectués :

(1) dans le cas intérieur pour $a_{0}=3 ; 4 ; e_{0}=0,4 ; 0,6 ; i_{0}=20,40,60,80,85^{\circ}$; $\omega_{0}=0,45,90^{\circ}$.

(2) dans le cas extérieur pour $a_{0}=8 ; 9 ; 10 ; e_{0}=0,4 ; 0,6 ; i_{0}=20,40,60,80^{\circ}$; $\omega_{0}=90^{\circ}$.

Des résultats partiels sont illustrés par les figures 1 à 5 . Les variations calculées de $\Omega(t)$ ne sont pas montrées car le système étudié possède la symétrie de révolution (orbite de $J$ supposée circulaire). On a déterminé la transition circulatoire-oscillatoire pour $\omega(t)$ qui, par example pour $a_{0}=4, e_{0}=0,4$ est comprise entre $i=20^{\circ}$ et $i=21^{\circ}$. Cette valeur de $i$ est voisine de celle obtenue par Kozai (1962) qui élimine les termes à courte période par la méthode de von Zeipel. Un résultat à noter consiste en la diminution sensible du semi-grand axe $a$, pour les grandes inclinaisons; dans ce cas, l'excentricité varie beaucoup et la distance périhélie $q$ étant très faible au voisinage de $e=1$, les termes de Poynting-Robertson deviennent importants. Dans ces conditions, la durée moyenne de vie physique de la particule météorique peut être réduite fortement alors que, dans la présente étude de dynamique, on ne considère que la durée de vie orbitale. Les approches avec Jupiter qui se produisent pour certaines conditions initiales nécessitent, pour leur exploitation, une étude plus complète qui est en cours.

\section{Remerciements}

L'auteur remercie M. J. Kovalevsky, du Bureau des Longitudes, Paris, pour l'intérêt constant qu'il porte à cette étude, et tient à exprimer sa vive gratitude à ses collègues du C.N.E.T. Lannion, MM le Clerc et Leguen, pour leur contribution essentielle à la programmation numérique.

\section{Références}

Halphen, G. H.: 1886, Traité des fonctions elliptiques, Paris, Tome I.

Hamming, R. W.: 1959, J. Assoc. Comput. Machinery 6, 37.

Kozai, Y.: 1962, Astron. J. 67, 591.

Musen, P.: 1963, Rev. Geophys. 1, 85.

Robertson, H. P.: 1937, Monthly Notices Roy. Astron. Soc. 97, 423.

Wyatt, S. P. et Whipple, F. L.: 1950, Astrophys. J. 111, 134.

\section{Discussion}

E.N. Kramer: In what way does consideration of the Poynting-Robertson effect influence the results?

J. Delcourt: For particles of magnitude 6-7 and for a period of $20000 \mathrm{yr}$ the PoyntingRobertson effect does not play an important part. But since we intend to increase the period of investigation the effect was taken into account in the equations. For smaller particles the effect would be of importance over the 20000 -yr period. 